\title{
105 Improvement of pad life in mirror polishing for GaAs wafers
}

\author{
Tetsuo OOKAWA, Takashi NISHIGUCHI \\ Hitachi, Ltd. Production Research Laboratory \\ Dept. of Process Technology Solutions \\ 292 Yoshida-cho Totsuka-ku Yokohama 2440817 Japan \\ E-mail address: ookawa.tetsuo@gm.perl.hitachi.co.jp
}

\begin{abstract}
Summary
GaAs wafers have contributed greatly to improve the performance of light-receiving and light-emitting devices. For such applications, a very low level of roughness, of the order of $0.1 \mathrm{nmRa}$, is required on GaAs wafers. If a polishing pad is used for a long time, the polishing rate decreases and the roughness on the wafer increases with increase of polishing time. In this study, the surface morphology of the polishing pad was focused as a dominant factor affecting the polishing rate, and, using an image processing technique, a quantitative approach was tried based on an optical microscope image. For the purpose of increasing the lifetime of polishing pads, a further study was made in which the polishing resistance, which correlates strongly with the polishing rate, was monitored.
\end{abstract}

Key words: GaAs wafer, polishing pad, polishing rate, surface morphology , micro-roughness, polishing resistance

\section{Introduction}

GaAs is one of the compound semiconductors which has better electrical and power saving properties than silicon. Compound semiconductors such as GaAs are widely used in opto-electronics and communications. In particular, in recent years, the demand for higher speed mobile communication has increased. GaAs wafers have contributed greatly to improve the performance of light-receiving and light-emitting devices, and a higher level of accuracy and quality is further required to achieve multi-functional and better performances in GaAs wafers.

For such applications, a very low level of roughness $0.1 \mathrm{nmRa}$, is required on $\mathrm{GaAs}$ wafers. If a polishing pad continues to be used for a long time, the polishing rate decreases and the roughness on the wafer increases. Therefore, although many studies have been done regarding the mirror polishing process for GaAs or an other wafers ${ }^{1-7}$, not enough has yet been done to verify the relationship between the surface morphology of the polishing pad and the polishing rate.

In GaAs wafer polishing, an oxide film is formed on the wafer due to a chemical reaction with the oxidants contained in the polishing liquid, and the mechanical polishing action follows on the oxide film. Because GaAs is a compound semiconductor, various types of oxide are formed. When a thicker oxide film is formed, the roughness increases? ${ }^{\text {) }}$. Therefore, it is supposed that the oxide film becomes thicker and the roughness of the wafer increases when the polishing rate decreases. In this study, the relationship among the thickness of the oxide film formed on the surface, the roughness and the polishing rate were examined in mirror polishing of GaAs wafers. According to the previous study ${ }^{8}$, the polishing pad becomes deformed and defaced with increasing polishing time, and thus the polishing rate of the film decreases. In this study, the surface morphology of the polishing pad was highlighted as a dominant factor affecting the polishing rate, and a quantitative approach using an image processing technique was tried based on an optical microscope image of polishing pad. For the purpose of increasing the lifetime of polishing pads, a further study was made in which the polishing resistance, which correlates strongly with the polishing rate, was monitored.

\section{Experiment \\ 2.1 Polishing experiment}

Fig. 1 shows a diagram of the polishing method and the polishing resistance measurement. Some wafers were polished simultaneously in a batch process. Table 1 shows the polishing conditions. A suede-type material was used as the polishing pad. The polishing liquid used was a chlorine oxidizing agent diluted with pure water (diluted by 50:1). Prior to the polishing experiments, the surface of a polishing pad was conditioned using a nylon brush dresser pushed on to the surface with dropping pure water as a conditioning liquid.

2.2 Evaluation of surface morphology of polishing pad

To pursue the reasons for the decrease in the polishing rate, an elemental analysis of the surface and the surface morphology of the polishing pad were evaluated before and after the polishing experiments. For the elemental analysis, an EDX element analyzer was used. Prior to the analysis, a 2-3nm thick layer of carbon was deposited on the pad surface for charge-up protection. ASEM and an optical microscope were used for evaluating the surface morphology.

To observe the polishing pad roughness with an optical microscope, white light shone down on the pad surface and the highest plateaus were observed at a magnification of 50. Also, the image contrast was enhanced using PC and a surface 


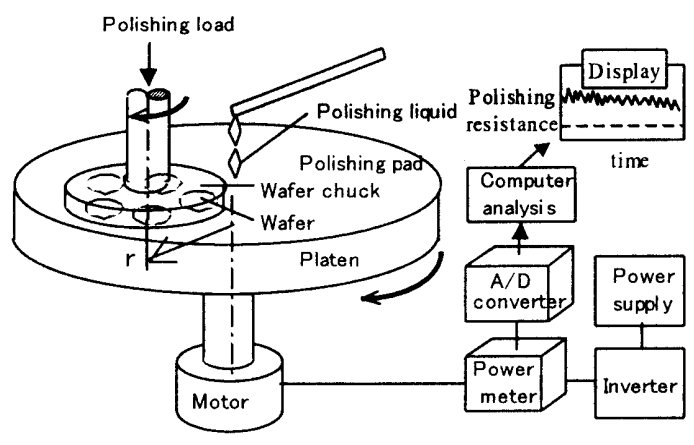

Fig.1 Diagr am of the polishing method and the polishing resistance measurement
Table1 Polishing conditions

\begin{tabular}{|l|l|}
\hline Wafer & $100 \mathrm{~mm} \mathrm{GaAs,crysal} \mathrm{orient} \mathrm{at} \mathrm{ion(100}$ \\
Polishing pad & Swede-type polish ing pad \\
Polishing liquid & Sodium hypoch lorite water solution \\
& $(294 \mathrm{~K})$ \\
Platen diamet er & $1270 \mathrm{~mm}$ \\
Platen speed & $45 \mathrm{~min}^{-1}$ \\
Polishing pressure & $5 \mathrm{kPa}$ \\
Thickness & Laser thickness measurement, \\
measurement & 5 point measurement in average \\
Interrupt ingtime & of polishing pad \\
Conditioning tool & $1-200$ min \\
Conditioning liquid & Nylon blush (prior to polishing) \\
\hline
\end{tabular}

Fig. 2 AFM images of GaAs wafer surface

morphology was displayed in binary form, with only the highest plateaus displayed as white points on a black screen. The area ratio of the highest plateaus to the whole surface image was calculated.

\subsection{Evaluation of oxide film thickness on wafer surface}

The oxide film thickness on the wafer surface was measured using a spectroellipsometer. In order to examine the formation of the oxide film derived from the chemical reaction to the polishing liquid, the growing rate of the oxide film on a wafer was measured at various dipping times in the polishing liquid.

\subsection{Evaluation of micro-roughness of wafer surface}

The micro-roughness, $\mathrm{Ra}$, on a wafer surface was measured using an AFM (Atomic Force Microscope) and the correlation with the oxide film thickness was examined.

\subsection{Measurement of polishing resistance}

A power meter was installed between the inverter power supply and the drive motor of the platen as shown in Fig. 1. A voltage signal in proportion to the power was detected to transfer to PC via an AVD converter. The polishing resistance was evaluated based on the voltage signal measured in the process.

The polishing resistance was calculated from:

$$
F_{t}=W /(2 \pi x N)
$$

where, $F_{t}$ : tangential component of force $(\mathrm{kN}), W$ : net power consumption $(\mathrm{kW}), r$ : distance $(\mathrm{m})$ between the centers of the platen and the wafer chuck, $N$ : Rotational speed of the platen $\left(\mathrm{s}^{-1}\right)$

The polishing speed and resistance were different every minute and every place on the wafer surface, because multiple wafers were polished simultaneously under planetary motion. In this experiment, the polishing resistance was represented at a distance of $400 \mathrm{~mm}$ from the center of the platen. In order to examine the polishing resistance change when running the polishing pad continuously, started a new polishing pad every polishing experiment. 


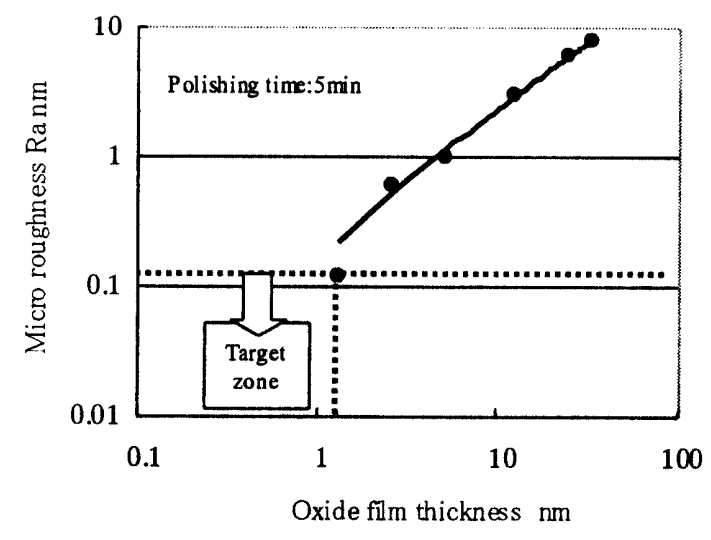

Fig.3 Correlation between oxide film thickness and surface roughness

\section{Experimental results and discussion}

3.1 Relationship between polishing rate and oxide film thickness on wafer

Micro-roughness on a wafer was measured at higher and lower polishing rate, which polishing amount was same. At a polishing pressure $5 \mathrm{kPa}$, Fig. 2 shows examples of AFM image of GaAs wafer surface. The polishing rate was $0.50 \mu \mathrm{m} / \mathrm{min}$ at $30 \mathrm{~min}$ polishing, and the micro-roughness was $0.13 \mathrm{~nm} \mathrm{Ra}$, while the polishing rate decreased to $0.21 \mu \mathrm{m} / \mathrm{min}$ at $440 \mathrm{~min}$ polishing, and the micro-roughness increased to $7.8 \mathrm{~nm}$ Ra. Fig. 3 shows the relationship between the oxide film thickness and the micro-roughness. The thicker the oxide film grows, the rougher the wafer surface becomes. According to the figure, an oxide film thickness has to be depressed less than 1.3-1.5nm, when a micro-roughness of $0.1-0.15 \mathrm{~nm}$ Ra is required on GaAs wafers.

Wafers were polished at different polishing rates, that is, under various polishing loads, and then the oxide films formed on the polished surfaces were measured. Fig. 4 shows the relationship between the polishing rate and the oxide film thickness. An oxide film of approximately $1.5 \mathrm{~nm}$ thick is formed on sample wafers before the experiment. The oxide film thickness after polishing is more likely to become thicker as the polishing rate is reduced. At a polishing rate of more than $0.4 \mu \mathrm{m} / \mathrm{min}$, the oxide film thickness is approximately $1.5 \mathrm{~nm}$, while thicker oxide film of $2-13 \mathrm{~nm}$ is found at polishing rates of less than $0.2 \mu \mathrm{m} / \mathrm{min}$. This seems to be explained in the following assumption, regard to the fact that the relationship between a polishing rate and a growing rate of the oxide film is in trade off. At a low polishing rate, it would be too slow to remove mechanically the oxide film exposing to be formed chemically in polishing liquid.

At a polishing rate of $0.20 \mu \mathrm{m} / \mathrm{min}$, the oxide film thickness is various in the wide range of $2-13 \mathrm{~nm}$. Thus, owing to the lower polishing load, there is inefficient or loose contact between the polishing pad and the wafer which makes the polishing rate differ around the wafer. This can also explain the reason for the scattered oxide film thickness obtained when the polishing rate was $0.01 \mu \mathrm{m} / \mathrm{min}$. It can be said that a polishing rate of more than $0.40 \mu \mathrm{m} / \mathrm{min}$ is required to keep the oxide film thickness formed on the wafer surface at approximately $1.5 \mathrm{~nm}$ using this type of polishing liquid.

Fig. 5 shows the growing rate of the oxide film when the wafer was dipped in the polishing liquid. It is considered that an oxide film containing various mixtures such as $\mathrm{Ga}_{2} \mathrm{O}_{3}, \mathrm{Ga}_{2} \mathrm{O}_{5}, \mathrm{As}_{2} \mathrm{O}_{3}, \mathrm{As}_{2} \mathrm{O}_{5}$ is formed on the wafer surface. Along with the dipping time, growing rate of the oxide film is reduced. This is because the oxidants in the liquid layer adjacent to the water surface become less active as the oxide film becomes thicker. At high polishing rates, the oxide film is mostly substantially removed and becomes thin just after it is formed. When the polishing pad continues to being used for a long time and the polishing rate is reduced, the oxide film grows thicker and the micro-roughness increases as the oxide film 


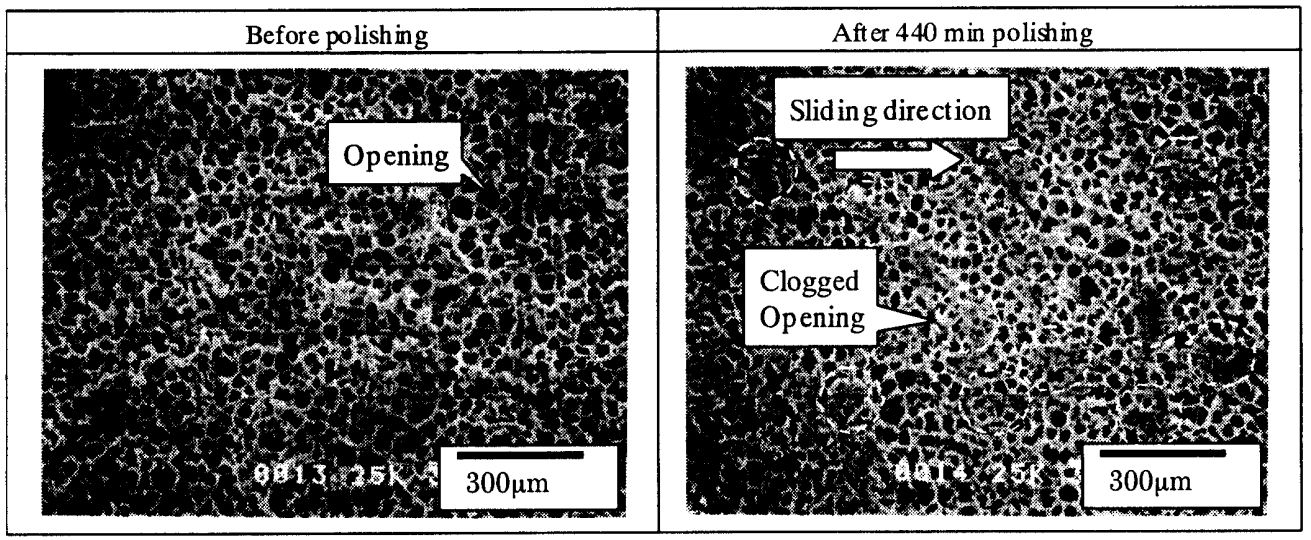

Fig6 SEM images of polishing pad surface

forming on the wafer surface is hardly removed.

\subsection{Morphology of polishing pad surface}

The surface morphology of a polishing pad of which the polishing rate was decreased was observed microscopically. The decrease in the polishing rate may be derived from deformation of the polishing pad. Fig. 6 shows SEM images of the polishing pad surface before and after polishing. The polishing rate after $440 \mathrm{~min}$ polishing decreased to about $60 \%$ compared to that with a fresh polishing pad. Many peaks were observed on the polishing pad at the start, while after 440 min polishing, many clogged openings in the sliding direction on the polishing pad surface were observed.

During polishing, the openings become deformed due to the polishing force and the polishing liquid is squeezed from the openings to the interface with the wafer. After the wafer moves off, the openings are again filled with polishing liquid. For continuous polishing, it may be that the polishing rate is decrease by the openings on the polishing pad getting clogged and the amount of polishing liquid in the openings becoming smaller.

An EDX element analysis was made to clarify either reason for the reducing polishing rate;

1. Plastic deformation of the pad, or

2. Pad openings become clogged with GaAs particles during polishing

The results show that some elements of the polishing pad material (polyurethane and others) and contamination such as $\mathrm{C}$, $\mathrm{O}, \mathrm{Al}$ were detected on pads before and after polishing. However, since neither Ga nor As were detected, it cannot be concluded that the openings of the polishing pad were filled with GaAs particles. Instead of this, the pad surface seems to be deformed by the force applied to the wafer during polishing.

The surface morphology of polishing pad was observed using an optical microscope as a non-contact, non-destructive method. Fig. 7 shows examples of micro photos of polishing surfaces. The upper images are focused on the highest regions. The lower images show the image contrast enhanced by taking a wider perspective image into PC. The total white area ratio relative to the whole area was $0.2 \%$ before polishing, and $1.1 \%$ after $440 \mathrm{~min}$ polishing. A significant difference was observed. Thus, the area ratio measured using the optical microscope image enables to give a quantitative evaluation of the flat area of the polishing pad surface.

\section{Polishing resistance}

A polishing pad recovers elastically after polishing, therefore, the wafer surface morphology during polishing is different from that after polishing. The polishing resistance was measured because it seems to have a strong correlation with the polishing rate. A interrupting polishing experiment was conducted at verious interrupting intervals so that polishing was restarted after some elastic recovery of the polishing pad. Fig. 8 shows an example of the polishing resistance. The abscissa shows the accumulated polishing time not including the interrupting interval and the ordinate shows the polishing resistance and pad interrupting interval. The polishing resistance decreases slightly as the polishing time increases. The polishing resistance decreases to $0.67 \mathrm{kN}$ after $440 \mathrm{~min}$ polishing, and by approximately $0.05 \mathrm{kN}$ for $200 \mathrm{~min}$. For a long interruption, the polishing resistance reclains to increase. The low polishing resistance is induced at a smaller polishing load for polishing time between 80 and $100 \mathrm{~min}$.

Fig. 9 shows the correlation between the polishing resistance and polishing rate. The polishing rate decreases as the polishing resistance decreases. The polishing rates decrease $1.6 \mu \mathrm{m} / \mathrm{min}$ per $\mathrm{kN}$. The polishing resistance needs to be more than $0.72 \mathrm{kN}$, as shown in Fig. 9, in order to achieve the desired polishing rate of more than $0.4 \mu \mathrm{m} / \mathrm{min}$ in Fig. 4 . As 


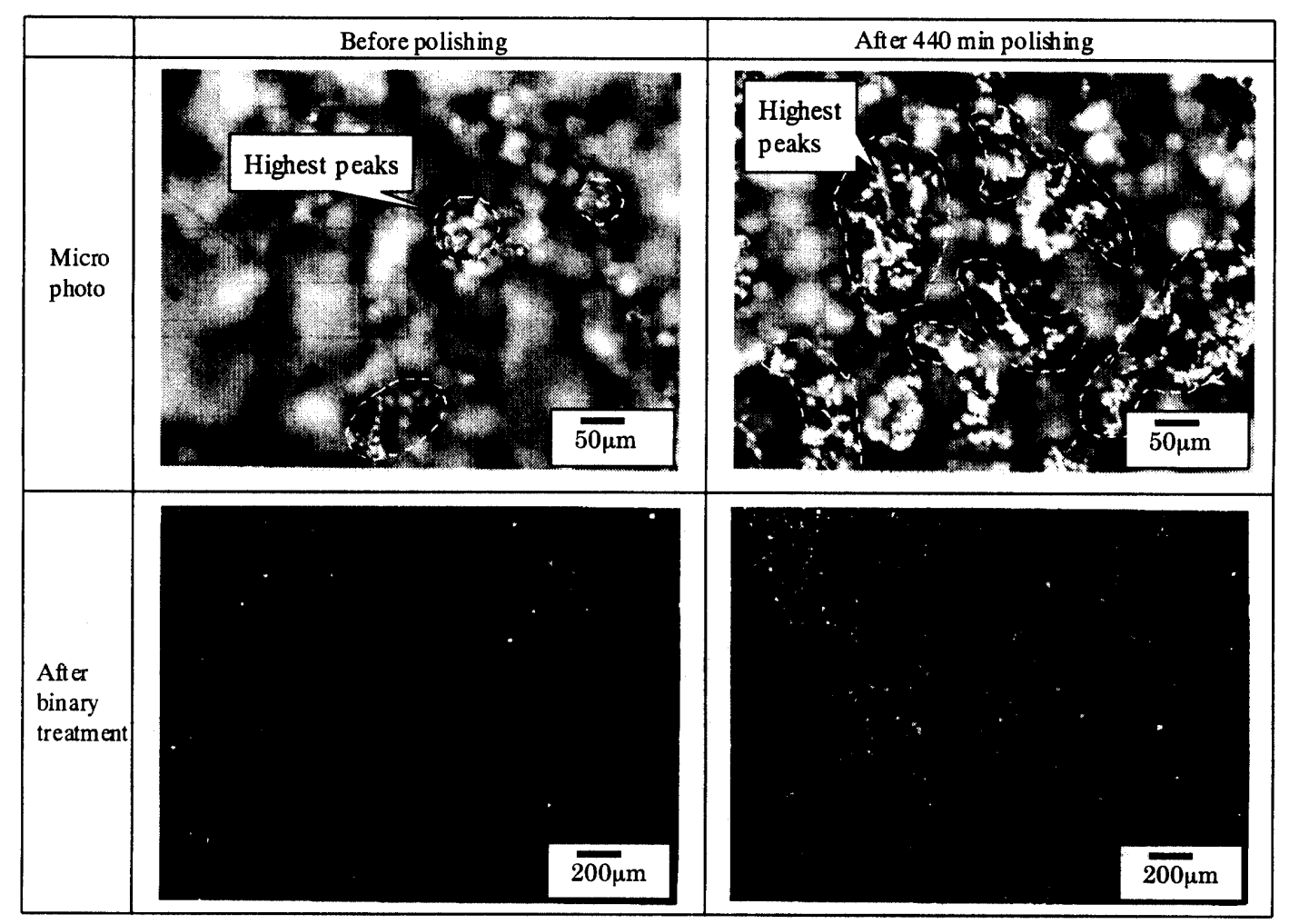

Fig 7 Polishing pad surface and black and white image at highest regions

shown in Fig. 8, a polishing time of $250 \mathrm{~min}$ at less than the target value of the polishing resistance is defined as the pad lifetime when continuous polishing is applied.

Hence, a longer pad life seems to be achieved by that polishing was interrupted before the polishing resistance reaches $0.72 \mathrm{kN}$, and restarts after recovery of the pad from deformation. Fig. 10 shows the experimental results on the lifetime of the pad under maintaining a high polishing resistance. The polishing resistance slightly increased after an interrupting interval 20-40 min; however, the polishing resistance recovers remarkably after an interruption of more than $180 \mathrm{~min}$.

When the polishing pad surface that was compressed to deform during polishing, is covered with water during interrupting to follow the elastic recovery gradually, the polishing pad swells and the openings are filled with water. It seems that the polishing resistance increases.

Such chemo-mechanical properties are subject to the creep recovery and water-absorbing characteristics common to all polishing pads made of polyurethane foam. It is estimated that the polishing resistance varies depending on the level of cross-linking and the amount of hardening agent in the pad. The significant increase in polishing resistance after an interrupting interval of more than $180 \mathrm{~min}$ is derived from the characteristics of the pad. For urethane with a higher level of cross-linking, the interrupting interval to come up with the same increase in polishing resistance is much longer due to the fact that water molecules can not infiltrate so easily into the polymerized urethane network. According to this experiment, it was possible to prevent the polishing resistance from continuously decreasing by having interrupting interval of more than $180 \mathrm{~min}$ every $100-200 \mathrm{~min}$ polishing. By repeating this interrupting cycle, the lifetime of the polishing pad increase by a factor of three, up to $750 \mathrm{~min}$ for interruptive polishing in comparison with $250 \mathrm{~min}$ for non-interruptive polishing. As wafer mounting time is longer than polishing time, the machine idle time were used for the interruption interval. Fig. 11 shows the correlation between the polishing pad interrupting interval and the recovery of the polishing resistance. The reason there were deviations in recovering the polishing resistance depends on some variations in the polishing resistance just before interruption. Taking these variations into consideration, it is concluded that the interrupting interval in the polishing pad requires $180 \mathrm{~min}$ to increase the polishing resistance by $50 \mathrm{~N}$ after $200 \mathrm{~min}$. In the experiment the maximum polishing time was $750 \mathrm{~min}$, however, the further lifetime of the pad is expected by optimizing the balance between the polishing and interrupting interval. 


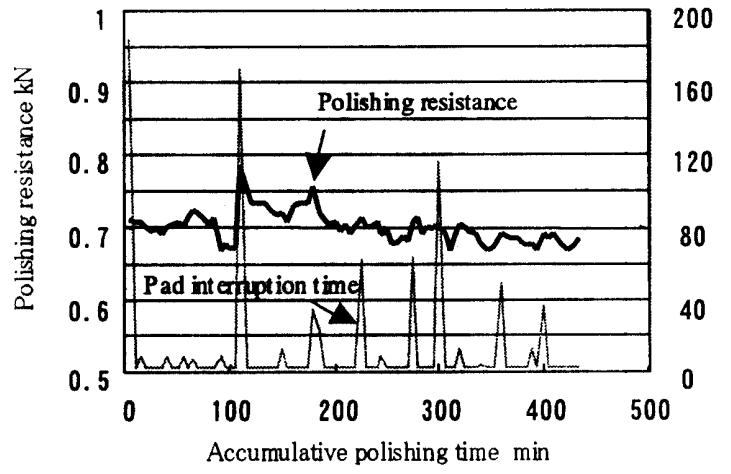

Fig.8 Change of polishing resistance

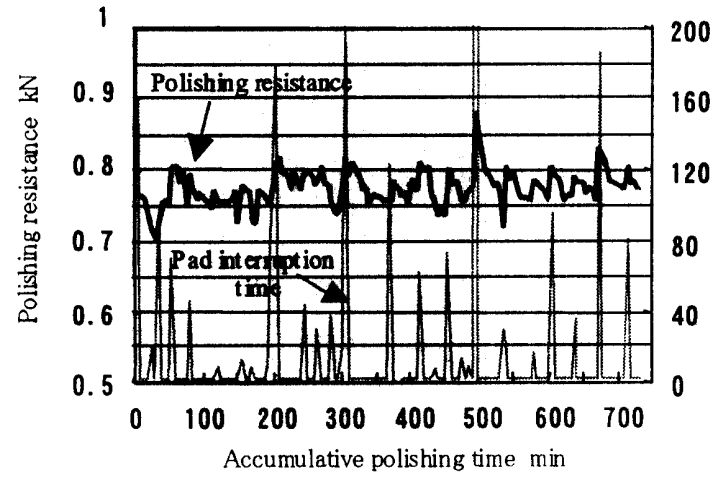

Fig.10 Lifetime of pad under maintaining the polishing resistance

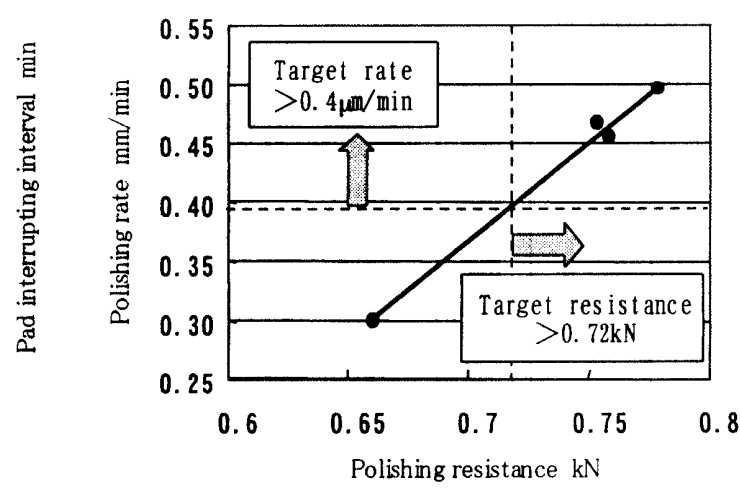

Fig.9 Correlation between polishing resistance and polishing rate

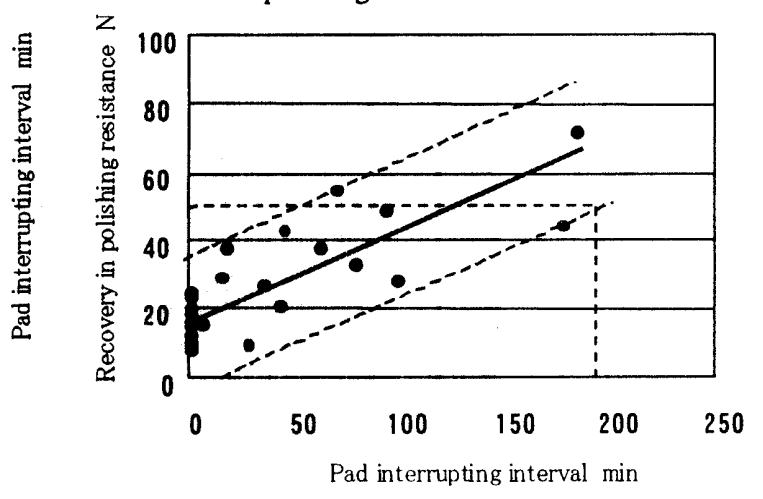

Fig.11 Correlation between pad interrupting interval and recovery of polishing resistance

\section{Conclusions}

In this paper, the fundamental polishing process for producing a mirror finish was examined on GaAs wafers using soft polishing pads. It is concluded as follows:

(1) It is verified through the experiments that the decrease in polishing rate during continuous polishing is due to the polishing pad surface becoming locally flat owing to plastic deformation.

(2) According to an image processing with an optical microscope, the white area ratio on the polishing pad surface with a less polishing rate is higher than that on a fresh polishing pad.

(3) When the polishing rate decreases, the polishing resistance also decreases. The ratio of the polishing resistance relative to the polishing rate is $1.6 \mathrm{um} / \mathrm{min} / \mathrm{kN}$ for the polishing pads worn-out by long use.

(4) By swelling a polishing pad in water, thus facilitating elastic recovery of the polishing pad, the polishing resistance is prevented from decreasing continuously. The lifetime of polishing pad, that is, accumulative polishing time, is achieved by a factor of three on the interrupting polishing process.

\section{References:}

1. T. Kasai, et al:: Precision Machining of Opto-electronic Crystals, Annals of the CIRP, 22,11(1973) 107-108.

2. S. Liyama: Damage Free Polishing of InP Substrates, Bull, Japan Soc. of Proc. Eng., 17, 4(1983) 285-289.

3. N.J.Brown: Some speculations on the mechanisms of abrasive grinding and polishing, Precision Eng., $9(1987)$ 129-138.

4. T. Kasai, et al.: Improvement of Conventional Polishing Conditions for Obtaining Super Smooth Surfaces of Glass and Metal Works, Annals of the CIRP, 39,1(1990) 321-324.

5. H. Akiyama, et al.: Improvement of GaAs Mirror Wafer Process for IC, Proc. Soc. Grind. Eng. (in Japanese) (1994) 35-38.

6. T. KARAKI-DOY, et al.: A New Processing Technique of GaAs Single Crystals and Its Mechanism, Int. J. Japan Soc. Proc. Eng., 30, 1(1996) 16-22.

7. T. Kasai, et al.: Newly Developed Automatic Polishing Machines for Obtainable Super-Smooth Surfaces of Compound Semiconductor Wafers, Annals of the CIRP, 37,1 (1988) 537-540.

8. T. Ookawa: Planarization of Interlayer Films by CMP, J. Soc. Grind. Eng.(in Japanese) 42, 11(1998) 463-466. 